\title{
Self-Doping of Ultrathin Insulating Films by Transition Metal Atoms
}

\author{
Z. Li, ${ }^{1}$ H.-Y. T. Chen, ${ }^{2}$ K. Schouteden, ${ }^{1}$ K. Lauwaet,,${ }^{1, *}$ L. Giordano, ${ }^{2}$ M. I. Trioni, ${ }^{3}$ E. Janssens, ${ }^{1}$ \\ V. Iancu, ${ }^{1}$ C. Van Haesendonck, ${ }^{1}$ P. Lievens, ${ }^{1, \dagger}$ and G. Pacchioni ${ }^{2,}$ \\ ${ }^{1}$ Laboratory of Solid-State Physics and Magnetism, KU Leuven, BE-3001 Leuven, Belgium \\ ${ }^{2}$ Dipartimento di Scienza dei Materiali, Università di Milano-Bicocca, Via Cozzi 53, I-20125 Milano, Italy \\ ${ }^{3}$ CNR-National Research Council of Italy, ISTM, Via Golgi 19, I-20133 Milano, Italy
}

(Received 18 October 2013; published 16 January 2014)

\begin{abstract}
Single magnetic $\mathrm{Co}$ atoms are deposited on atomically thin $\mathrm{NaCl}$ films on $\mathrm{Au}(111)$. Two different adsorption sites are revealed by high-resolution scanning tunneling microscopy (STM), i.e., at Na and at $\mathrm{Cl}$ locations. Using density functional based simulations of the STM images, we show that the Co atoms substitute with either a $\mathrm{Na}$ or $\mathrm{Cl}$ atom of the $\mathrm{NaCl}$ surface, resulting in cationic and anionic Co dopants with a high thermal stability. The dependence of the magnetic coupling between neighboring Co atoms on their separation is investigated via spatially resolved measurement of the local density of states.
\end{abstract}

DOI: 10.1103/PhysRevLett.112.026102

Deposition of metal atoms on solid surfaces is usually employed to study nucleation and growth of metal nanoparticles that are relevant for heterogeneous catalysis, sensors, optical and magnetic data storage, etc. Growth of ultrathin films of insulating materials on a metallic substrate prior atom deposition can provide additional control of the coupling of the metal adsorbates with the metallic substrate [1-3]. Soft landing of metal atoms on insulating thin layers is typically noninvasive and results in cluster growth with little or no surface damage. Alternatively, incorporation of the deposited atoms into an insulating material may lead to material doping and the possibility to tune catalytic, optical, magnetic, and transport properties of materials [4]. Examples of penetration of single adatoms into insulating thin films have been reported for the special case of porous networks such as $\mathrm{SiO}_{2}$ layers on Mo [5].

Investigation of individual magnetic atoms obviously requires a local probing technique with high spatial and energy resolution, a technique that is provided by scanning tunneling microscopy (STM) and scanning tunneling spectroscopy (STS). Electronic and magnetic properties of single atoms have been explored via Kondo effect measurements [6], inelastic electron tunneling spectroscopy [7], and resonant tunneling through the (unoccupied) energy levels of the atoms [8].

Here, relying on unoccupied electron resonant tunneling, we demonstrate that single $\mathrm{Co}$ atoms deposited on $\mathrm{NaCl}(100) / \mathrm{Au}(111)$ exhibit very different electronic behavior depending on their adsorption sites. We show that the $\mathrm{Co}$ atoms are incorporated into the $\mathrm{NaCl}$ surface by substituting either a $\mathrm{Na}$ atom or a $\mathrm{Cl}$ atom, resulting in cationic and anionic Co dopants, respectively. By mapping the local density of states (LDOS), we obtain indirect evidence about the magnetic coupling in Co dimers with different interatomic distance.
PACS numbers: 68.37.Ef, 68.55.Ln, 71.15.Mb, 73.61.Ng

All experiments are conducted in an ultrahigh vacuum system that includes a low temperature STM (Omicron Nanotechnology). Monolayer (1L) and bilayer (2L) $\mathrm{NaCl}(100)$ islands on $\mathrm{Au}(111)$ are obtained by deposition of $\mathrm{NaCl}(99.999 \%$ purity, Alfa Aesar) at $800 \mathrm{~K}$ on cold (well below room temperature) and on room temperature (RT) $\mathrm{Au}(111)$ surfaces, respectively. Trilayer (3L) $\mathrm{NaCl}$ is obtained by mild postannealing to about $470 \mathrm{~K}[9,10]$. Co atoms are electron-beam evaporated from a highly pure Co $(99.9996 \%$ purity, Alfa Aesar) rod. $(d I / d V)(V)$ curves are acquired with an open or closed feedback loop by lockin detection using amplitudes of 30 to $60 \mathrm{mV}$ at $800 \mathrm{~Hz}$. Maps of the LDOS are acquired with a closed feedback loop with similar parameter settings. All STM measurements are carried out at $4.5 \mathrm{~K}$ using a tungsten tip. Image processing is performed by Nanotec WSxM [11].

Density functional theory (DFT) calculations are performed using the generalized gradient approximation (GGA, Perdew-Burke-Ernzerhof functional [12]) and the plane waves code VASP [13,14]. The interaction between the ions and the valence electrons is described by the projector augmented wave method [15]. To take into account the lattice mismatch and the different symmetry of the film with respect to the substrate we constructed a model obtained by superposing a $(2 \times 2) \mathrm{NaCl}$ unit cell on a $\left(\begin{array}{ll}3 & 1 \\ 1 & 3\end{array}\right)$ superstructure of $\mathrm{Au}(111)$ [9]. STM images are generated in the Tersoff-Hamann approximation [16] using a $(4 \times 4)$ supercell. Dispersion interactions have been included with a pair-wise force field (DFT-D2 method of Grimme) [17].

Figure 1(a) presents an atomic resolution image of a $2 \mathrm{~L}$ $\mathrm{NaCl}$ island on $\mathrm{Au}(111)$ after deposition of $\mathrm{Co}$ atoms on the cold substrate (Co atoms are also deposited on $1 \mathrm{~L}$ and $3 \mathrm{~L}$ $\mathrm{NaCl}$ islands, see Supplemental Material [18]). Note that only the $\mathrm{Cl}$ atoms of the $\mathrm{NaCl}$ surface are resolved as 


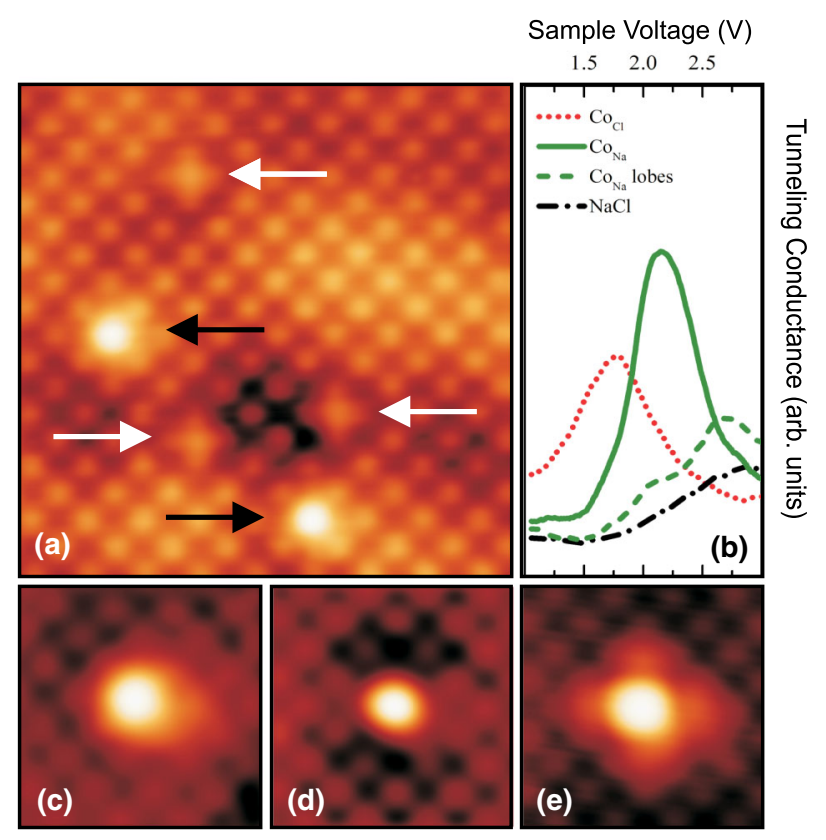

FIG. 1 (color online). (a) STM topography image of 5 Co atoms on $2 \mathrm{~L} \mathrm{NaCl} / \mathrm{Au}(111)$. Two "types" of $\mathrm{Co}$ atoms are resolved, i.e., at $\mathrm{Cl}$ locations $\left(\mathrm{Co}_{\mathrm{Cl}}\right.$, indicated by the black arrows) and at $\mathrm{Na}$ locations ( $\mathrm{CO}_{\mathrm{Na}}$, indicated by the white arrows). Image size is $5 \times 5 \mathrm{~nm}^{2}(V=+50 \mathrm{mV}, I=0.3 \mathrm{nA})$. (b) $d I / d V$ spectra recorded at $\mathrm{Co}_{\mathrm{Cl}}, \mathrm{Co}_{\mathrm{Na}}$ and the lobes around $\mathrm{Co}_{\mathrm{Na}}$. (c) $\mathrm{STM}$ topography image of $\mathrm{Co}_{\mathrm{Cl}}$ recorded at $+1.9 \mathrm{~V}$. Image size is $2.3 \times 2.3 \mathrm{~nm}^{2}(I=50 \mathrm{pA})$. (d) and (e) STM topography images of $\mathrm{Co}_{\mathrm{Na}}$ recorded at $+2.2 \mathrm{~V}$ and $+2.7 \mathrm{~V}$, respectively. Image sizes are $2.3 \times 2.3 \mathrm{~nm}^{2}(I=20 \mathrm{pA})$.

protrusions in the STM topography image [19]. Two different "types" of Co atoms can be retrieved. One type [black arrow] appears on the atomic rows, i.e., at the location of a $\mathrm{Cl}$ atom. This type is hereafter referred to as $\mathrm{Co}_{\mathrm{Cl}}$. The other type [white arrow] appears in between the atomic rows, i.e., at the location of a $\mathrm{Na}$ atom, and has a lower apparent height that is comparable to the corrugation of the $\mathrm{NaCl}$ surface. This type is hereafter referred to as $\mathrm{Co}_{\mathrm{Na}}$. Within the accuracy of our experiments, we did not observe a clear influence of the moiré and/or herringbone superlattice on the adsorption behavior of Co atoms (see Fig. S2 in Supplemental Material [18]). The amount of $\mathrm{Co}_{\mathrm{Na}}$ atoms is higher than that of $\mathrm{Co}_{\mathrm{Cl}}$ atoms, and the ratio is not affected by postannealing the sample up to RT. In contrast, upon deposition on a RT substrate, only $\mathrm{Co}_{\mathrm{Na}}$ is retrieved. This behavior differs from that of $\mathrm{Ag}$ and $\mathrm{Au}$ atoms on $2 \mathrm{~L} \mathrm{NaCl}$ on $\mathrm{Cu}(111)$ [1,20], for which only one adsorption site (on top of $\mathrm{Cl}$ atoms) was reported.

Figures 1(b)-1(e) present dI/dV data and STM topography images of $\mathrm{Co}$ atoms on $2 \mathrm{~L} \mathrm{NaCl} / \mathrm{Au}(111)$. The $\mathrm{Co}_{\mathrm{Cl}}$ atoms exhibit a broad resonance state in the unoccupied LDOS around $1.8 \mathrm{eV}$, while the $\mathrm{Co}_{\mathrm{Na}}$ atoms are characterized by a main resonance state around $2.2 \mathrm{eV}$ and a small shoulder at around $2.7 \mathrm{eV}(d I / d V$ taken on the center of $\mathrm{Co}_{\mathrm{Na}}$ ). The resonance difference between $\mathrm{Co}_{\mathrm{Cl}}$ and $\mathrm{Co}_{\mathrm{Na}}$ may be related to a different number of electrons on the Co atoms; i.e., the charge transfer between the $\mathrm{Co}$ and the $\mathrm{NaCl}$ film differs for the two adsorption sites. Both $\mathrm{Co}_{\mathrm{Cl}}$ and $\mathrm{Co}_{\mathrm{Na}}$ atoms appear more pronounced in STM topography images recorded at voltages corresponding to the resonance state when compared to lower voltages [compare Fig. 1(a) to Figs. 1(c) and 1(d), respectively]. Moreover, $\mathrm{CO}_{\mathrm{Na}}$ atoms exhibit four extra lobes at voltages corresponding to the shoulder around $2.7 \mathrm{~V}$, as illustrated in Fig. 1(e). This shoulder becomes a main resonance in STS spectra recorded at the center of such a lobe [see Fig. 1(b)]. The resonances in Fig. 1(b) can be related to the tunneling through the unoccupied $3 d$ states of the Co atoms (see text below). STS analysis of the $\mathrm{NaCl} / \mathrm{Au}(111)$ interface state indicates that the $\mathrm{Co}$ atoms are located at the $\mathrm{NaCl}$ surface and not at the $\mathrm{NaCl} / \mathrm{Au}(111)$ interface (see Fig. S4 in Supplemental Material [18]).

Several adsorption sites were considered in DFT calculations of supported and unsupported $2 \mathrm{~L}$ and $3 \mathrm{~L} \mathrm{NaCl}$ films (see Supplemental Material [18] for details). In the following, we focus on $2 \mathrm{~L} \mathrm{NaCl}$. We find that a Co atom has such a high affinity for the $\mathrm{NaCl}$ film that it takes the position of a $\mathrm{Na}$ atom in the top layer by substitutional doping with an adsorption energy of almost $3 \mathrm{eV}$, thereby pushing $\mathrm{Na}$ in an interstitial site. $\mathrm{Cl}$ substitution in the top layer is energetically less favorable than $\mathrm{Na}$ substitution: removing the $\mathrm{Co}$ atom from a $\mathrm{Cl}$ substitutional position costs $1.3 \mathrm{eV}$ while removing the $\mathrm{Co}$ atom from a Na substitutional position costs $5.1 \mathrm{eV}$. While the $\mathrm{Na}$ atom moves to interstitial sites and then to the Au substrate (both processes are thermodynamically favorable), the displaced $\mathrm{Cl}$ atom does not diffuse to the interface nor does it tend to desorb as $1 / 2 \mathrm{Cl}_{2}$ (thermodynamically unfavorable processes). However, it is likely that the replaced $\mathrm{Cl}$ atom, and possibly also the replaced $\mathrm{Na}$ atom, are displaced to the borders of the $\mathrm{NaCl}$ islands. These diffusion processes imply small energy barriers (not investigated in detail) that can be easily overcome by the energy released in the adsorption process. In both cases, the resulting $\mathrm{NaCl}$ film is basically undistorted after substitution of $\mathrm{Na}$ or $\mathrm{Cl}$ [structural models in Figs. 2(a) and 2(b), respectively].

Next, we analyzed the electronic properties of the two different adsorption sites. A free Co atom has a $4 s^{2} 3 d^{7}$ configuration. When it replaces a $\mathrm{Na}^{+}$or $\mathrm{Cl}^{-}$ion in the $\mathrm{NaCl}$ lattice, the Bader charge is $+0.9 \mathrm{e}$ or $-0.4 \mathrm{e}$, respectively, which indicates it takes +1 or -1 formal charge. The electronic configurations become $4 s^{0} 3 d^{8}$ (Co replacing $\mathrm{Na}$, $\mathrm{Co}_{\mathrm{Na}}$ ) and $4 s^{2} 3 d^{8}$ (Co replacing $\mathrm{Cl}, \mathrm{Co}_{\mathrm{Cl}}$ ). The magnetic moment is in both cases $2.3 \mu_{\mathrm{B}}$ (five $3 d_{\alpha}$ and three $3 d_{\beta}$ electrons). This can also be observed in the projected density of state (PDOS) spectra in Fig. 2. Below $\mathrm{E}_{\mathrm{F}}$, one can identify 5 occupied $3 d_{\alpha}$ and 3 occupied $3 d_{\beta}$ states (see also Fig. S6 in Supplemental Material [18]). For Co substituting $\mathrm{Cl}$, the Co $4 s p$ states can be observed as well below $\mathrm{E}_{\mathrm{F}}$. 


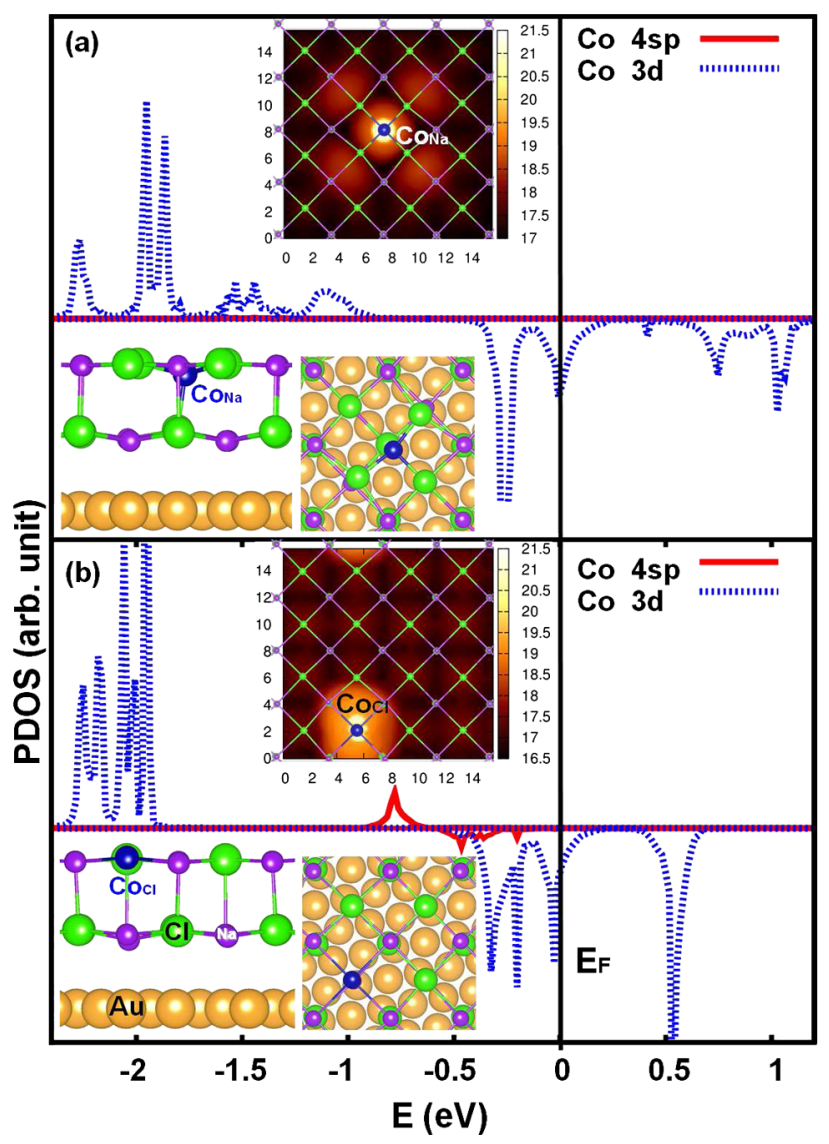

FIG. 2 (color online). Projected density of states (PDOS) of $2 \mathrm{~L}$ $\mathrm{NaCl} / \mathrm{Au}(111)$ films with $\mathrm{Co}$ substituting $\mathrm{Na}$ (a) and $\mathrm{Cl}$ (b), respectively. See Fig. S6 in Supplemental Material [18] for the decomposition of Co $3 d$ orbitals and $4 s p$ orbitals. Structures and simulated STM images are shown in the insets.

Above $\mathrm{E}_{\mathrm{F}}$, for $\mathrm{Co}_{\mathrm{Cl}}$ the two $3 d_{\beta}$ orbitals appear at the same energy [Fig. 2(b)], while for $\mathrm{Co}_{\mathrm{Na}}$, they are well separated [Fig. 2(a)].

Note that the empty states appear at lower energies when compared to the experiment, i.e., around 0.75 and $1.0 \mathrm{eV}$ for $\mathrm{Co}_{\mathrm{Na}}$ [Fig. 2(a)] and around $0.5 \mathrm{eV}$ for $\mathrm{Co}_{\mathrm{Cl}}$ [Fig. 2(b)]. This is not surprising since the $\mathrm{NaCl}$ band gap $(9 \mathrm{eV})$ is considerably underestimated by about $4 \mathrm{eV}$ by DFT-GGA calculations. This is also the case for localized d states of transition metal atoms where the separation between filled and empty $3 d$ states is typically underestimated in standard DFT calculations. This discrepancy does, however, not affect the interpretation of our experiments. The PDOS curves in Figs. 2(a) and 2(b) reveal that the empty Co $3 d$ states for $\mathrm{Co}_{\mathrm{Cl}}$ appear at a lower energy $(0.25$ and $0.5 \mathrm{eV})$ when compared to $\mathrm{Co}_{\mathrm{Na}}$, in agreement with the experimental observations [Fig. 1(b)].

More direct evidence that unoccupied d orbitals are probed in the experiment and that Co atoms take substitutional positions in the $\mathrm{NaCl}$ lattice comes from simulated STM topography images (integrated LDOS over the unoccupied $3 d$ states) [see the upper insets in Figs. 2(a) and 2(b)]. For Co substituting $\mathrm{Na}$, the lower Co empty state has a $d_{z^{2}}$ character which protrudes towards the vacuum, giving rise to an intense and symmetric image, in agreement with Fig. 1(d). Differently, the peak at higher energy is the in-plane $d_{x y}$ orbital and it is responsible for the four lobes structure that is observed experimentally [Fig. 1(e)]. These four lobes arise because the $\mathrm{Cl}$ atoms close to $\mathrm{CO}_{\mathrm{Na}}$ move towards the Co impurity. This is accompanied by a hybridization of the Co $3 d$ empty states with the $\mathrm{Cl} 3 p$ orbitals, resulting in a higher isodensity around the $\mathrm{Cl}$ atoms (see Fig. S7 in the Supplemental Material [18]) and, consequently, in a brighter appearance in the simulated STM image. Overall, the simulated STM images in Fig. 2 are in excellent agreement with the experiment.

Since a single $\mathrm{Co}$ atom replacing $\mathrm{Na}$ has a $3 d^{8}$ configuration with two unpaired electrons, Co dimers represent an ideal system to study magnetic interactions between diluted magnetic impurities in an insulating matrix. The total wave function of the magnetic dimer can be described as the product of a spatial and a spin part. Concerning the latter part, the exchange interaction between the two atoms of the dimer splits the $3 d$ orbital related unoccupied resonance into a pair of states, of which one state exhibits a symmetric character and the other state exhibits an antisymmetric character. Following the Pauli exclusion principle, the total wave function of the dimer must be antisymmetric with respect to exchange of any two identical electrons (holes), e.g., that of the $3 d$ orbital related resonance states. This implies that when the spin wave function is symmetric the spatial part must be antisymmetric and vice versa, which can be visualized in the LDOS maps.

Figures 3(a) and 3(b) present $d I / d V$ spectra of two types of Co dimers with Co-Co separations of $0.40 \mathrm{~nm}$ (dimer I) and $0.56 \mathrm{~nm}$ (dimer II). These distances correspond exactly to Co atoms occupying nearest and next nearest neighbor Na lattice sites. For dimer I, the $d I / d V$ spectra reveal that the exchange interaction has split the $3 d$ orbital related resonance into a low-energy state at $1.63 \mathrm{eV}$ and a highenergy state at $1.9 \mathrm{eV}$ [Fig. 3(a)]. Lee et al. [8] previously showed that the unoccupied spin split $s$ and $p$ states of magnetic dimers yield information about the internal magnetic coupling (that is governed by $\mathrm{d}$ states) between the atoms of the dimer. In particular, by comparing with the theoretical LDOS curves, they found a higher $d I / d V$ intensity at the center of magnetic dimers for resonances of symmetric character, while the $d I / d V$ intensity was highest at both ends of the dimer for resonances of antisymmetric character. The characters of the $3 d$ orbital related resonances of the Co dimer in Fig. 3(a) can be directly visualized in LDOS maps recorded at the corresponding tunneling voltage [21]: the nodal plane between the two Co atoms in Fig. 3(c) (enclosed by the dashed ellipses) reflects the antisymmetric character of the 
Sample Voltage (V)

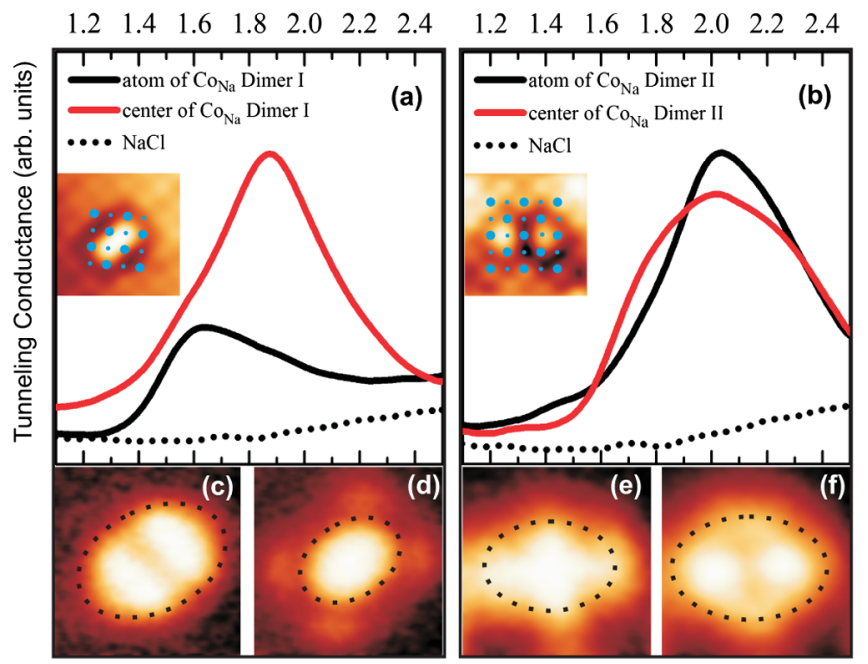

FIG. 3 (color online). (a) $d I / d V$ spectra of Co Dimer I. (b) $d I / d V$ spectra of Co Dimer II. Insets in (a) and (b) present atomic resolution STM topography images (taken at $1.0 \mathrm{~V}$ ) of the two types of $\mathrm{Co}$ dimers with schematic $\mathrm{NaCl}$ lattice (bigger circles represent $\mathrm{Cl}$ atoms and smaller ones $\mathrm{Na}$ atoms) on top. (c) and (d) $2 \times 2 \mathrm{~nm}^{2}$ LDOS images of Dimer I taken at 1.6 and $1.9 \mathrm{~V}$. (e) and (f) $2 \times 2 \mathrm{~nm}^{2}$ LDOS images of Dimer II taken at $1.8 \mathrm{~V}$ and $2.0 \mathrm{eV}$.

low-energy state $\left(E_{\text {anti }}^{I}\right)$, while the maximum intensity between the two $\mathrm{Co}$ atoms in Fig. 3(d) reflects the symmetric character of the high-energy state $\left(E_{\text {sym }}^{I}\right)$. As discussed above, this implies that the corresponding spin combination of unoccupied $3 d$ states of dimer I is symmetric for the low-energy state and antisymmetric for the high-energy state.

For dimer II, the energy splitting due to the spin exchange interaction is too small to be distinguished in the spectra and only a broad peak is observed [Fig. 3(b)]. However, we can resolve two split states in the LDOS maps. Figures 3(e) and 3(f) present the low-energy state at $1.8 \mathrm{eV}\left(E_{\mathrm{sym}}^{I I}\right)$ with a symmetric character and the highenergy state at $2.0 \mathrm{eV}\left(E_{\text {anti }}^{I I}\right)$ with an antisymmetric character. This indicates that the corresponding spin combination of unoccupied $3 d$ states of dimer II is antisymmetric for the low-energy state and symmetric for the high-energy state. Such a distance dependence of the spatial symmetry character of unoccupied resonances can only occur for magnetic dimers. Previously, it was shown by Nilius et al. for the case of nonmagnetic $\mathrm{Au}$ dimers that the spatial symmetric (antisymmetric) resonance always has the low (high) energy independent of the $\mathrm{Au}-\mathrm{Au}$ separation distance [21].

Next, we analyze the $d I / d V$ data using the Heisenberg model. If we define the spins of the two unoccupied states of the atoms constituting the dimer as $\mathbf{S}_{1}$ and $\mathbf{S}_{2}$ and ignore possible superexchange via the $\mathrm{NaCl}$ lattice, the Heisenberg exchange Hamiltonian can be written as $\mathbf{H}_{\mathrm{ex}}=J \mathbf{S}_{1} \cdot \mathbf{S}_{2}$ where $J$ is the spin coupling constant of the unoccupied states: $J<0$ indicates a ferromagnetic (FM) coupling and $J>0$ indicates an antiferromagnetic (AFM) coupling. Since both atoms have the same spin value $S_{1}=S_{2}=s$, the difference between the eigenvalues of the spin symmetric state $\left(S_{\mathrm{sym}}=S_{1}+S_{2}=2 \mathrm{~s}\right.$, corresponding to the spatial antisymmetric state, $E_{\text {anti }}$, and the spin antisymmetric state $\left(S_{\text {anti }}=S_{1}-S_{2}=0\right.$, corresponding to the spatial symmetric state, $\left.E_{\mathrm{sym}}\right)$ is $\Delta E=E_{\text {anti }}-E_{\text {sym }}=$ $J s(2 s+1)[22]$.

Using the formal spin of the embedded $\mathrm{Co}^{+}$atom $(s=1)$ and the measured exchange splitting yields $J<0$ and $J>0$ for dimer I and dimer II, respectively. Note that the sign of $J$, and hence, the FM or AFM character, depends on the assignment of the exchange-split resonance states, while it does not depend on the exact value of the spin $s$. Because the spin up (down) of the unoccupied $3 d$ state is accompanied by a spin down (up) of the occupied $3 d$ state, the spin coupling of the unoccupied $3 d$ state should be the same as that of the occupied states, as illustrated in Fig. 4. This indicates that the occupied $3 d$ states for dimer I and dimer II also experience FM and AFM coupling, respectively. Similar distance-dependent FM and AFM magnetic couplings have been reported for $\mathrm{Co}$ dimers on $\mathrm{Cu}(100)[6,23]$ and for $\mathrm{Gd}$ dimers on $\mathrm{CuN}$ [24]. The magnetic coupling between the Co atoms in a Co dimer is, apart from the interatomic separation, expected to depend on the occupation sites of the dimer atoms. However, dimers other than $\mathrm{Co}_{\mathrm{Na}}-\mathrm{Co}_{\mathrm{Na}}$ were not retrieved in our experiments.

To summarize, in a combined study of STM experiments and DFT based simulations we demonstrated that evaporated $\mathrm{Co}$ atoms interact strongly with a $2 \mathrm{~L}$ $\mathrm{NaCl}(100) / \mathrm{Au}(111)$ support and incorporate into the $\mathrm{NaCl}$ film replacing either $\mathrm{Na}$ or $\mathrm{Cl}$ ions. This is a rather uncommon example of spontaneous doping as transition metal atoms deposited on thin insulating films usually
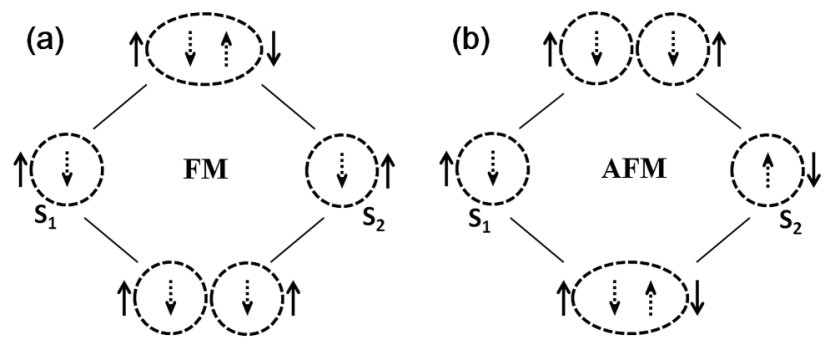

FIG. 4. Schematic illustration of the magnetic interactions between the unoccupied $3 d$ states in magnetic dimers for the case of (a) ferromagnetic and (b) antiferromagnetic coupling between the unoccupied states of the individual atoms. The solid arrows are total spin labels for the occupied states, while the dashed circles and arrows represent the spatial wave functions and total spins of the unoccupied states, respectively. Following the Pauli exclusion principle, the spin and spatial wave functions exhibit opposite symmetry. 
experience adsorption, surface diffusion, and cluster growth. This system turns out to be ideal for studying magnetic interactions between Co ions. Direct probing and manipulation of the magnetic coupling for embedded Co dimers using spin polarized STM experiments are envisaged.

This research has been supported by the Research Foundation-Flanders (FWO, Belgium), the Flemish Concerted Action (Project No. GOA/09/006) research program, and the Italian MIUR (FIRB Project No. RBAP115AYN). Z. L. thanks the China Scholarship Council for financial support (No. 2011624021). K. L. acknowledges the Institute for the Promotion of Innovation through Science and Technology in Flanders (IWTVlaanderen). The authors thank D. A. Muzychenko for useful discussions. K. S. and V. I. acknowledge support from the FWO.

*Present address: IMDEA Nanociencia, C/Faraday, 9, Campus Universitario de Cantoblanco, 28049 Madrid, Spain. peter.lievens@fys.kuleuven.be ¥gianfranco.pacchioni@unimib.it

[1] J. Repp, G. Meyer, F. E. Olsson, and M. Persson, Science 305, 493 (2004).

[2] J. Repp, G. Meyer, S. Paavilainen, F. E. Olsson, and M. Persson, Phys. Rev. Lett. 95, 225503 (2005).

[3] C. F. Hirjibehedin, C.-Y. Lin, A. F. Otte, M. Ternes, C. P. Lutz, B. A. Jones, and A. J. Heinrich, Science 317, 1199 (2007).

[4] E. W. McFarland and H. Metiu, Chem. Rev. 113, 4391 (2013).

[5] M. Baron, D. Stacchiola, S. Ulrich, N. Nilius, S. Shaikhutdinov, H. J. Freund, U. Martinez, L. Giordano, and G. Pacchioni, J. Phys. Chem. C 112, 3405 (2008).

[6] P. Wahl, P. Simon, L. Diekhöner, V. S. Stepanyuk, P. Bruno, M. A. Schneider, and K. Kern, Phys. Rev. Lett. 98, 056601 (2007).
[7] C. F. Hirjibehedin, C. P. Lutz, and A. J. Heinrich, Science 312, 1021 (2006).

[8] H. J. Lee, W. Ho, and M. Persson, Phys. Rev. Lett. 92, 186802 (2004).

[9] K. Lauwaet, K. Schouteden, E. Janssens, C. Van Haesendonck, P. Lievens, M. I. Trioni, L. Giordano, and G. Pacchioni, Phys. Rev. B 85, 245440 (2012).

[10] K. Lauwaet, K. Schouteden, E. Janssens, C. Van Haesendonck, and P. Lievens, J. Phys. Condens. Matter 24, 475507 (2012).

[11] I. Horcas, R. Fernández, J. M. Gómez-Rodríguez, J. Colchero, J. Gómez-Herrero, and A. M. Baro, Rev. Sci. Instrum. 78, 013705 (2007).

[12] J. P. Perdew, K. Burke, and M. Ernzerhof, Phys. Rev. Lett. 77, 3865 (1996).

[13] G. Kresse and J. Furthmüller, Comput. Mater. Sci. 6, 15 (1996).

[14] G. Kresse and J. Furthmüller, Phys. Rev. B 54, 11169 (1996).

[15] G. Kresse and D. Joubert, Phys. Rev. B 59, 1758 (1999).

[16] J. Tersoff and D. R. Hamann, Phys. Rev. B 31, 805 (1985).

[17] S. Grimme, J. Comput. Chem. 27, 1787 (2006).

[18] See Supplemental Material at http://link.aps.org/ supplemental/10.1103/PhysRevLett.112.026102 for more details about the STM experiments and DFT calculations.

[19] W. Hebenstreit, J. Redinger, Z. Horozova, M. Schmid, R. Podloucky, and P. Varga, Surf. Sci. 424, L321 (1999).

[20] F. E. Olsson, S. Paavilainen, M. Persson, J. Repp, and G. Meyer, Phys. Rev. Lett. 98, 176803 (2007).

[21] N. Nilius, T. M. Wallis, M. Persson, and W. Ho, Phys. Rev. Lett. 90, 196103 (2003).

[22] C. J. Slater, J. Phys. Colloq. 33, C3-7 (1972).

[23] V.S. Stepanyuk, A. N. Baranov, D. I. Bazhanov, W. Hergert, and A. A. Katsnelson, Surf. Sci. 482-485, 1045 (2001).

[24] C.-Y. Lin, J.-L. Li, Y.-H. Hsieh, K.-L. Ou, and B. A. Jones, Phys. Rev. X 2, 021012 (2012). 